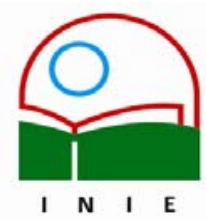

Actualidades Investigativas en Educación

Revista Electrónica publicada por el

Instituto de Investigación en Educación

Universidad de Costa Rica

ISSN 1409-4703

http://revista.inie.ucr.ac.cr

COSTA RICA

\title{
CONTRIBUCIONES AL ESTUDIO DE LA FORMACIÓN ACADÉMICA DE LA UNIVERSIDAD DE COSTA RICA: SUS VÍNCULOS CON LOS PROBLEMAS SOCIALES Y LA POBREZA
}

CONTRIBUTIONS TO THE STUDY OF ACADEMIC EDUCATION FOR THE UNIVERSITY OF COSTA RICA: ITS LINKS WITH SOCIAL PROBLEMS AND POVERTY

\author{
Volumen 8, Número 3 \\ pp. 1-22
}

Este número se publicó el 15 de diciembre 2008

Freddy Esquivel Corella

La revista está indexada en los directorios:

LATINDEX, REDALYC, IRESIE, CLASE, DIALNET, DOAJ, E-REVIST@S,

La revista está incluida en los sitios:

REDIE, RINACE, OEI, MAESTROTECA, HUASCARAN 


\title{
CONTRIBUCIONES AL ESTUDIO DE LA FORMACIÓN ACADÉMICA DE LA UNIVERSIDAD DE COSTA RICA: SUS VÍNCULOS CON LOS PROBLEMAS SOCIALES Y LA POBREZA

\author{
CONTRIBUTIONS TO THE STUDY OF ACADEMIC EDUCATION FOR THE UNIVERSITY \\ OF COSTA RICA: ITS LINKS WITH SOCIAL PROBLEMS AND POVERTY
}

\section{Freddy Esquivel Corella}

\begin{abstract}
Resumen: El presente artículo, resultado parcial de un estudio más amplio, problematiza la importancia que adquieren los problemas sociales (y en especial la pobreza), en la formación académica de la Universidad de Costa Rica. Los datos se derivan de un total de 292 encuestas (mediante un muestreo por conveniencia), que se aplicaron a estudiantes de carreras de las facultades de Educación, Ciencias Sociales, Derecho y Ciencias Económicas, así como a 41 docentes de la misma universidad. Entre los principales hallazgos se encuentra que el tema de los problemas sociales, y en especial la pobreza, se constituye en un eje de interés para los y las estudiantes, aunque en sus planes de estudio parece no ser un contenido relevante. Tal situación puede no ser coherente con los fines y principios que orientan a la Universidad, sin diferencia del campo de conocimiento de cada carrera. A su vez, el contenido expuesto destaca la necesidad de establecer un programa, línea o núcleo de investigación que genere insumos sobre las relaciones entre la educación universitaria y la "cuestión social", como forma de articularse con los currículos de las diferentes carreras.
\end{abstract}

Palabras claves: FORMACIÓN ACADÉMICA, PROBLEMAS SOCIALES, POBREZA, UNIVERSIDAD DE COSTA RICA.

\begin{abstract}
This article, a result in part of a larger study, problematized the importance of social problems (especially poverty), in formal education at the University of Costa Rica. The data are derived from a total of 292 surveys, which were applied to students for careers in the faculties of Education, Social Sciences, Law and Economics, as well as 41 teachers from the same university. Among the major findings is that the theme of social problems, especially poverty, is an axis of interest to students and, although in their curricula do not seem to be a relevant content. Such a situation may not be consistent with the purposes and principles that guide the University, not unlike the area of knowledge. In turn, explained the content stresses the need to establish a program that generates inputs on the relationship between university education and social problems as a way to link up with the curricula of different races.
\end{abstract}

Key words: EDUCATION, SOCIAL PROBLEMS, POVERTY, UNIVERSIDAD DE COSTA RICA.

\begin{abstract}
1 Doctor en Educación de la Universidad Estatal a Distancia (SEPUNED). Magíster Scientiae en Trabajo Social y Licenciado en Trabajo Social ambos títulos de la Universidad de Costa Rica. Profesor e investigador de la Escuela de Trabajo Social (UCR), Maestría en Trabajo Social (UCR) y Maestría Centroamericana en Estudios Interdisciplinarios en Discapacidad (UCR). Autor de los libros: Gerencia Social: un análisis crítico desde el Trabajo Social (2005) Buenos Aires, Argentina: Espacio Editorial. Introducción al Trabajo Social: Una breve guía conceptual y contextual (2006). San José. Costa Rica: EUCR. Trabajo Social en Costa Rica. Del ideario liberal a su constitución en el reformismo. (2007). San José, Costa Rica: EUCR. Autor de alrededor de quince artículos sobre Trabajo Social, movimientos sociales y gerencia social.
\end{abstract}

Dirección electrónica: freddy.faessa@gmail.com

Artículo recibido: 4 de setiembre, 2008

Aprobado: 4 de diciembre, 2008 


\section{1- Introducción}

El presente artículo tiene como propósito contribuir al estudio de la formación académica de la Universidad de Costa Rica (UCR), especialmente, desde el compromiso de aportar a la búsqueda de la justicia social y el combate a la miseria, como dos de sus finalidades institucionales ${ }^{2}$.

Los insumos que se exponen a continuación, forman parte de un estudio más amplio que se aproxima al tema de las tensiones entre la formación que se gesta en dicha casa de enseñanza y la base orgánico institucional que la constituye (Ver Esquivel, 2007).

A partir de la fuente antes citada es poco frecuente hallar pesquisas que coloquen el tema de la pobreza como un objeto de interlocución con la formación académica, a pesar de que el contexto contemporáneo costarricense y centroamericano, se caracterizado como afirma Rojas (2005) por la disparidad de ingresos entre las distintas fracciones de la población.

Por tanto, el tratar de aportar al debate de la formación académica universitaria, y las tensiones que presenta en relación con las devastaciones de la pobreza, se plantea con la finalidad de analizar su significado histórico y social, así como el significado que adquiere la educación superior pública.

Lo anterior se destaca, ya que en las sociedades capitalistas contemporáneas, la universidad pública se presenta ante desafíos no conocidos (Vargas, 2006).

A su vez, se recuerda que la institución universitaria pública, ha jugado un papel histórico en la sociedad moderna, ya que ella es síntesis de contradicciones, pero en esencia, es una entidad "formadora" para responder a las demandas de las relaciones sociales que caracterizan al modo de producción imperante y a las tensiones de clases que se contienen en su reproducción.

Por ello, se parte de que la formación académica está entramada en esas demandas que se le presentan a la Universidad, la cual, debe responder a las exigencias propias de las sociedades en que se desenvuelven.

Entre las condiciones que influyen en el caso particular de la Universidad de Costa Rica, se halla su surgimiento en un Estado que buscaba el enfrentamiento hacia la "cuestión

2 Dichas referencias se hallan contenidas en Estatuto Orgánico de la UCR, documento que ha orientado desde su fundación sus fines, organización, estructura más amplia y articulaciones. Se sugiere al lector consultar el título I, artículo 50, incisos b y d; artículo 52 incisos b,c,d,f; artículo 108; artículo 114 y artículo 184 de dicho documento oficial. 
social", desde el ideario liberal reformista ${ }^{3}$ (Salazar, 1995) y que se va transformando hasta el actual siglo XXI.

Dicha tendencia, junto con otras que se vincularon, produjeron en la UCR, una particular base orgánica, la cual, se ha contenido, pero a la vez, transformado a lo largo de las décadas.

A partir de ello, se encuentra un proyecto de Universidad que se singulariza por las contradicciones entre la formación, el mercado, la sociedad y el Estado.

Con estos aportes, como se ha recalcado, se pretende aportar al estudio de las contradicciones que se expresan en las históricas tensiones universidad-sociedad-mercado, contenidas en la formación académica universitaria, la cual tiene el deber de concatenarse con las orientaciones teleológicas que orienta su Estatuto Orgánico, ya resaltado líneas atrás.

Por ello, el análisis del vínculo de la formación académica universitaria y el tema de los problemas sociales y la pobreza, no se gesta como una relación casual, en un país determinado, este es un análisis ético-político inevitable para las instituciones educativas públicas.

\section{2- Cuestiones metodológicas del estudio:}

Metodológicamente cabe anotar que los datos estadísticos que se exponen en este artículo, se derivaron de 292 encuestas (mediante un muestreo por conveniencia ${ }^{4}$ ) realizadas a alumnos ${ }^{5}$ de diferentes carreras de las facultades de Educación, Ciencias Sociales,

${ }^{3}$ Un interesante análisis se encuentra en Solís (1992).

${ }^{4}$ A ello vale recordar que: En las muestras no probabilísticas, la elección de los elementos no dependen de la probabilidad sino de causas relacionadas con las características del investigador o del que hace la selección. Aquí el procedimiento no es mecánico ni con base en formulas de probabilidad, sino que depende del proceso. (Hernández et al., 1998, p. 2007-2008)

${ }^{5}$ Es importante aclarar que este artículo reconoce las diferencias de género en materia de producción de conocimiento, sin embargo, con el fin de poder hacer una exposición más fluída de los resultados únicamente se hará distinción entre hombres y mujeres cuando se considere necesario. Entre los rasgos básicos de la población de estudiantes se destaca: Las edades de un $58.90 \%$, se concentran entre los 20 y 23 años inclusive. Un 53\% del total encuestado son hombres y un $47 \%$ son mujeres. Las provincias donde viven, según porcentajes, son San José $(63.1 \%)$, Heredia $(13.7 \%)$, Cartago $(11.3 \%)$ y Alajuela $(10,9 \%)$ principalmente. Un $65 \%$ provienen de colegios públicos, un $19,8 \%$ de privados y un $14,7 \%$ de semiprivados o de otra naturaleza. El $69,9 \%$ afirma tener beca, de ellos el $40,4 \%$ destaca que es beca 11 , un $17,7 \%$ señala que su beca es 10 . Un $9,1 \%$ refiere a tener beca 9 ; los estudiantes con becas de estímulo y honor suman un $7 \%$. Por lo tanto, la población que se consultó en el estudio, tenían cierta trayectoria en la UCR, ya que no eran en su mayoría de ingreso reciente. 
Derecho y Ciencias Económicas, así como a 41 docentes ${ }^{6}$ de esta casa de enseñanza, específicamente sobre el tema de la formación académica y sus vinculaciones con la realidad costarricense.

Cabe citar que el período de recogida de la información se desarrolló durante un mes, consultando a estudiantes de diferentes carreras de las distintas Facultades citadas, el aparecimiento de datos de ciertas Escuelas o Departamentos y la no inclusión de otras fue resultado tanto por la naturaleza del muestro, como por la voluntad de colaborar en el trabajo de campo, así como los momentos en que se logró hacer la consulta con los recursos con que se contaba. Por otra parte, y según se evidencia en Esquivel (2007), la mayoría de las investigaciones sobre el tema de formación académica se centraban en el análisis de una única carrera, sin tampoco vincular su relación con el tema de los problemas sociales y la pobreza.

De tal manera, se buscó innovar en los puntos de partida para tratar el objeto, por lo que se identificaron las principales carreras (así como las Facultades a las que pertenecían) convocadas por la Vicerrectoría de Acción Social (llamada extensión universitaria en otros países) y la Sección de Trabajos Comunales Universitarios ${ }^{7}$ a la hora de desarrollar proyectos vinculados al tema de los problemas sociales ${ }^{8}$.

En síntesis un 32, 53\% eran de la Facultad de Ciencias Económicas, un 30,13\% de la Facultad de Educación, un $25,68 \%$ de la Facultad de Ciencias Sociales y finalmente un $11,66 \%$ de la Facultad de Derecho.

Tal elección tenía los siguientes propósitos: a) identificar las principales carreras y facultades que son llamadas para proyectos relacionados con cuestiones tan relevantes del

\footnotetext{
${ }^{6}$ La participación de los docentes de dichas carreras fue planteada en el diseño, pero a su vez se convirtió en una limitante para el estudio, debido a su poca colaboración, aunque un reducido número posibilitó aportes para el análisis.

${ }^{7}$ Toda persona que estudia en la UCR y que desee graduarse de Bachiller en cualquier área, debe cumplir con un total de 300 horas de trabajo comunal en el campo de su profesión ( en equipos interdisciplinarios), ello como retribución a la sociedad costarricense por subsidiar su educación superior.

${ }^{8}$ Entre ellos se citan: Análisis del riesgo por desastres y planificación urbana de los asentamientos humanos informarles del cantón Central de San José; Apoyo a la gestión comunal en seguridad alimentaria y nutricional en cantones prioritarios; Apoyo a mujeres trabajadoras sexuales; Apoyo al proyecto infantil, familia, salud y deporte en la Clínica de Pavas; Apoyo al proyecto de la Ciudad de los Niños; Capacitación en áreas educativo recreativas para albergues y centros de acogimiento familiar del PANI: niños, jóvenes y madres adolescentes; Fortalecimiento de la protección y asistencia de los refugiados y población migrante vulnerable en Costa Rica; Por el fortalecimiento del trabajo con la niñez y la adolescencia del cantón de Upala: conjunción de esfuerzos interinstitucionales y comunales. (Fuente: Guía de Matricula de TCU, I Ciclo, 2006)
} 
contexto nacional como la "cuestión social"; b) tener una muestra de estudiantes y docentes de diferentes carreras, con el fin de innovar en estudios de esta naturaleza, según se evidenció en los antecedentes consultados, donde los estudios se habían concentrado en una sola carrera; c) ampliar el ángulo de análisis del objeto, tanto en su heterogeneidad como en su complejidad.

La validación de la encuesta ${ }^{9}$ a los estudiantes universitarios, se realizó probando el instrumento con tres personas inscritas en Facultades que no se tenían entre las que iban a estudiarse (Medicina, Biología y Agronomía); cada vez que se hacía la validación se fueron realizando las correcciones del caso, hasta en la tercera vez en que fueron muy pocas las cuestiones por mejorar.

En el caso de las pruebas de los cuestionarios dirigidos a docentes, se recurrió a cuatro profesoras de otra sede de la UCR (Sede de Universitaria de Occidente, ubicada en la provincia de Alajuela) las cuales hicieron valiosos aportes a la corrección del mismo.

Como se detalla más adelante, los resultados se generaron por una escala de valoración (buena, regular, mala, muy mala y pésima) recurriendo a las definiciones de la Real Academia Española $(2001)^{10}$, y el contraste que se produjo en la prueba del instrumento.

El instrumento fue diseñado de manera general, de tal forma que la encuesta no se planteó para una carrera en específica, situación que como se ha señalado, no era el fin del trabajo, por tanto se trataba de tener una visión panorámica, ante una base formativa que se supone de igual orientación para todas las carreras, ello según se dicta en el Estatuto Orgánico (Ver primera nota al final del comento).

En lo que refiere al análisis, es importante destacar que este artículo se centra únicamente en la estadística descriptiva, ya que al ser parte de un estudio más amplio (que es imposible de integrar acá) no se integran acá el total de insumos y esfuerzos analíticos desarrollados, donde estos datos son básicamente un insumo contextual y de referencia.

\footnotetext{
${ }^{9}$ La estructura general del instrumento (similar en ambas poblaciones), se basó principalmente en la valoración general de: la formación de la UCR, el vínculo de esa formación con la realidad nacional, los cursos vinculados al estudio de la realidad nacional y la existencia de otros cursos vinculados al estudio de la realidad nacional en sus carreras.

10 Por buena se entiende "útil, con un propósito para algo"; por muy buena refiere a una "mayor utilidad y pro positividad para algo", por regular se comprende "de condición media o inferior a ella", por mala se define como "que carece de utilidad, o se aleja de su propósito", por muy mala se refiere a "una ausencia significativa de utilidad, o se distancia ampliamente de su propósito". Dichos términos, Volumen 8, Número 3, Año 2008, ISSN 1409-4703
} 


\section{3- Algunas referencias previas}

A partir del estudio de Esquivel (2007), y en el total de las personas consultadas (sin clasificarlas según carrera) fue posible identificar que la valoración general de la formación académica universitaria en la UCR, según la muestra de estudiantes y docentes, se ubica como "muy buena".

A pesar de la valoración predominante, se pudo tener evidencia de que la misma no es homogénea, ni se localiza por igual en todos los escenarios académicos de la muestra, ello, ya que se hallan diferencias entre facultades, y en las facultades entre sus carreras.

Por otra parte, se indagó sobre la valoración que daban estudiantes y docentes al vínculo formación académica-contexto nacional; las encuestas de los y las estudiantes arrojaron un mayor porcentaje a la casilla de "buena" (37,67\%); en el caso de los docentes, tal vínculo lo valoraron como "muy bueno" (51\%), sin embargo, el porcentaje es menor si se le compara con el dato de la estimación en general de la formación que obtuvo un $61 \%$, y que en el estudiantado bajo esta última casilla reunía un 43,83\%.

En la investigación citada, también se realizó una serie de entrevistas a las autoridades universitarias (Rectora, Vicerrectoras y Vicerrectores, Miembros del Consejo Universitario), en su discurso, fue recurrente el referir al Sistema de Educación General como el espacio por excelencia para que los y las estudiantes se "sensibilicen" con la Costa Rica de hoy.

Aún así, entre las personas entrevistadas se plantean preocupaciones tales como, que se ha perdido la esencia de los pensadores que crearon lo que hoy se conoce como Sistema de Educación General ${ }^{11}$, así como el desafío de profundizar sobre los fines y principios de la Universidad de Costa Rica.

Por tanto, en la encuesta realizada a los y las estudiantes, se preguntó por la valorización de los cursos de Estudios Generales y Seminarios de Realidad Nacional (pertenecientes al Sistema de Educación General); los resultados arrojan que un 26\% los califican como "buenos", 23.3\% "regulares", 15.1\% "excelentes" y 12.7\% "pésimos"; el restante $2.7 \%$ "no sabía o no respondía".

como cualquier otra valoración, se colocan siempre ante el desafío de la interpretación subjetiva de las personas que se consultan a la hora de aplicar las escalas valorativas (Elejabarrieta e Iñiguez, 1984)

${ }^{11}$ El Sistema de Educación General constituye una base formativa fundamental de la UCR que permite fortalecer la formación generalista, humanista e integral, en función de avanzar más allá de la mera especialización, y posibilitar el enriquecimiento académico de los y las estudiantes en función de la reflexión de las demandas contextuales y el significado de sus profesiones. 
A su vez, en los datos de los docentes, aunque predomina una "muy buena" valoración $(34,1 \%)$ de los cursos vinculados con la realidad nacional, los porcentajes son menores que los que se dieron en la relación de la formación con la realidad nacional (51\%); valga anotar que un $14 \%$ de los académicos no respondió o alegó no saber.

En términos generales y relacionando estos datos con los que refieren a la formación académica universitaria, los mismos tienen una menor valoración positiva, similar a lo que sucede cuando se cuestiona sobre la articulación de la formación con la realidad nacional.

Al parecer, dichos cursos son los que tienen menor valoración en la población encuestada; sin embargo, para las autoridades universitarias, son los más importantes para establecer ese nexo.

Tomando lo antes aportado, es posible que se puedan establecer tensiones entre las expectativas de las autoridades académicas de los cursos en mención, y su tarea de "catalizadores" de la sensibilidad profesional y social para los y las estudiantes.

En síntesis, se puede destacar que el tema de la relación formación académica universitaria y realidad nacional, genera una serie de interrogantes de relevancia, en especial cuando las coordenadas de análisis parten de los fines y principios que se establecen en el Estatuto Orgánico de la UCR.

Retomando los datos expuestos, también se consultó a los y las estudiantes sobre la existencia en sus planes de estudio sobre otras materias que establezcan el nexo de su formación con la realidad nacional; al respecto las respuestas, denotan que hay diferencias por Facultades y Carreras en lo que refiere a la presencia de más cursos vinculados al tema en cuestión, hallándose diferencias tales como que un $78.49 \%$ desconoce su existencia en Educación, o un 65,33\% que da una respuesta afirmativa en Ciencias Sociales.

Al incorporar los datos expuestos, se puede concluir que un $63 \%$ del total de estudiantes encuestados, alega que no recibe otras materias sobre realidad nacional, más allá que las obligatorias por la UCR en su Sistema de Educación General.

Para una importante cantidad de personas la base de estudio de la realidad nacional pareciera depender principalmente de esos cursos obligatorios, condición que realza la necesidad de establecer una interlocución crítica con este fundamento de la formación académica universitaria en la UCR.

Lo anterior, expresa tanto la importancia que adquiere el Sistema de Educación General en la formación, como a su vez, conduce a interrogarse sobre su significado para los y las estudiantes, ya que por su parte han sido los de menor "valoración positiva". 
Entonces, es evidente la existencia de contradicciones entre las apreciaciones de las autoridades universitarias, las valoraciones de los (as) estudiantes y, la de los docentes, en lo que a la vinculación de la formación académica con la realidad nacional refiere.

Con ese panorama, se procede a profundizar sobre la importancia que adquieren los problemas sociales, y en especial la pobreza, en la formación en cuestión.

\section{4- Los problemas sociales y la pobreza en la formación académica de la UCR}

Según las políticas institucionales de la UCR, la búsqueda de aportar a las transformaciones que la sociedad costarricense requiere, conlleva a que se genere una importante reflexión sobre las demandas de la realidad costarricense contemporánea y la formación académica.

Los llamados problemas sociales (desempleo, farmacodependencia, violencia, inseguridad, entre otros) son un desafío complejo en el actual contexto nacional; y sin duda, como se evidencia en diferentes estudios (tales como el Estado de la Nación -CONARE-, los Planes Nacionales de Desarrollo, y los Informes sobre Desarrollo Social -MIDEPLAN-, por ejemplo) la pobreza es un tema de relevancia nacional.

Los contenidos que continúan buscan generar algunas reflexiones sobre la importancia que podría o no tener la pobreza en algunas carreras de la UCR, así como su explicación por las personas entrevistadas. (De antemano cabe aclarar que no se brindó ninguna definición previa de pobreza a quienes contestaron la encuesta, por el contrario esa fue una interrogante incluida en el instrumento, que conllevó a identificar una importante asociación causal entre pobreza y ausencia de educación)

Entre las interrogantes del estudio realizado, se consultó a los estudiantes, si en sus planes de carrera (más allá de los impartidos por el Sistema de Educación General de la UCR) recibían cursos vinculados con problemas sociales, al respecto se obtuvieron los siguientes datos: 


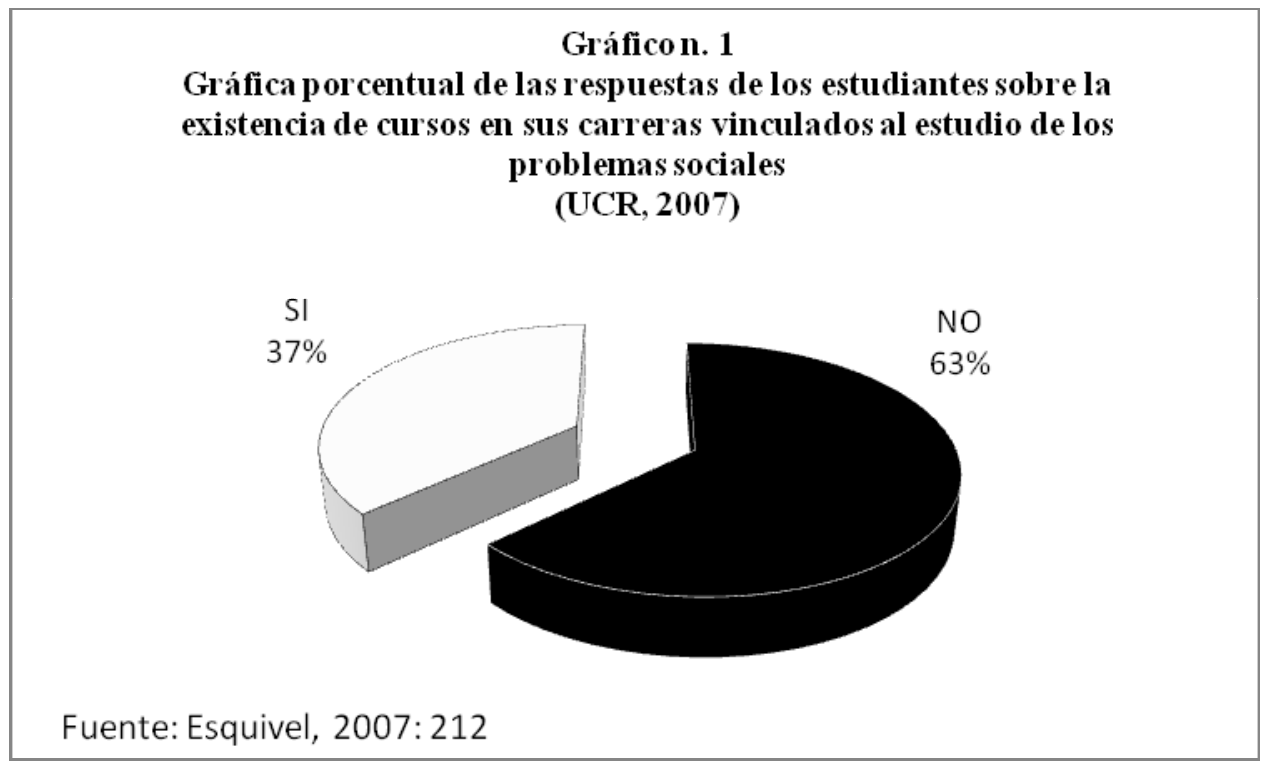

Por lo cual se puede estimar, que según los universitarios entrevistados, únicamente un $37 \%$, afirma la existencia de cursos vinculados al tema de los problemas sociales, más allá de los que se brindan en el Sistema de Educación General.

Desde otro ángulo, los docentes consultados de las diferentes escuelas, afirman que a lo largo de la formación universitaria, los problemas sociales adquieren una "buena" importancia, para ilustrar lo anterior se presenta el siguiente gráfico:

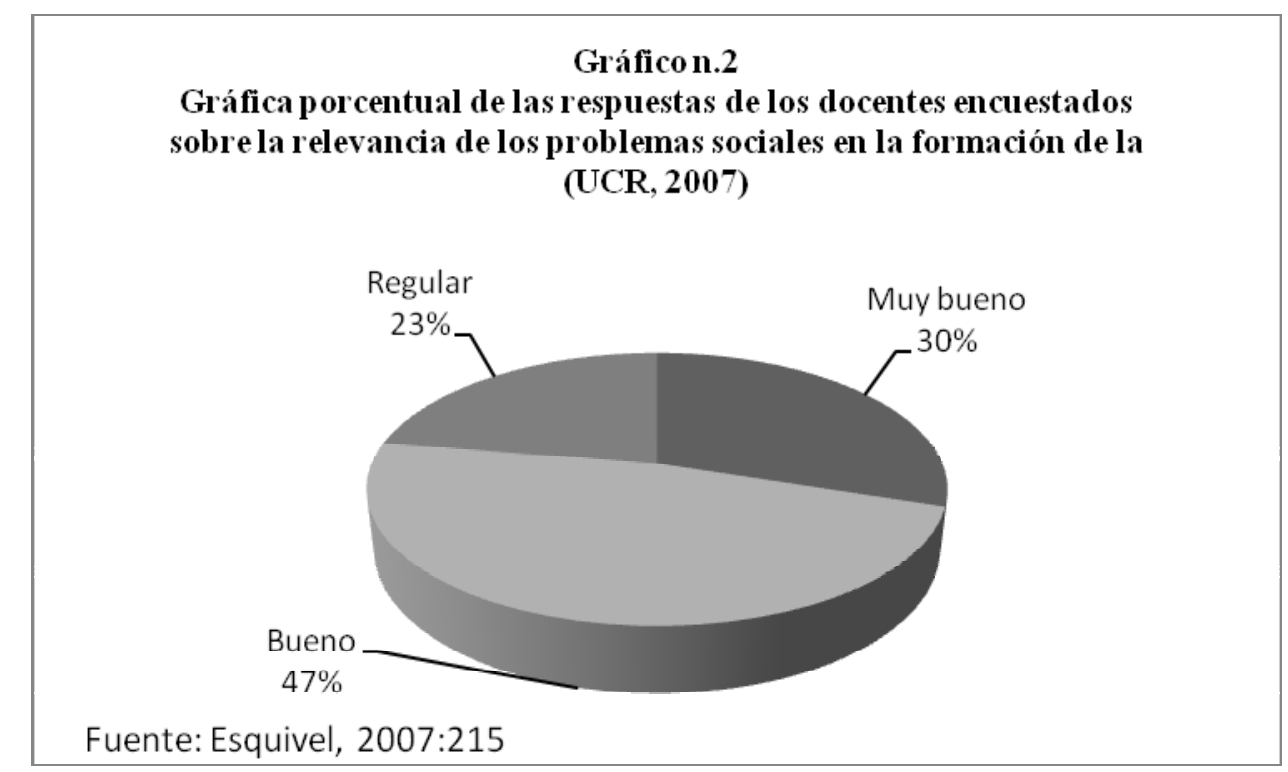


En síntesis, hay diferencias entre la relevancia que tienen los problemas sociales desde la opinión de los estudiantes, y la que expresan los docentes consultados.

Por otra parte, en este tema, el Sistema de Educación General de la UCR, adquiere un papel fundamental, en tanto es una plataforma que surge para lograr incorporar y problematizar esas bases de la formación universitaria de dicha casa de enseñanza, sin embargo, cabe interrogarse sobre la posibilidad de que ese análisis sea diluido por los planes de estudio de las carreras, las cuales podrían delegar a ese Sistema, una responsabilidad que según el Estatuto Orgánico de la UCR, compete a todas las carreras y todos sus cursos.

Vinculado a ello, se cuestionó de manera abierta a los estudiantes, sobre los principales problemas sociales de interés según su formación. Los siguientes cuadros, resumen algunos considerados como prioritarios para su formación académica, según carreras y facultades:

\section{Cuadro $N^{\circ} 1$}

Principales problemas sociales de importancia para la formación, según los estudiantes encuestados según carrera.

Facultad de Ciencias Sociales UCR, 2007

\begin{tabular}{|c|l|}
\hline Carrera & \multicolumn{1}{c|}{ Información básica } \\
\hline Antropología & Pobreza, violencia, inseguridad y desempleo. \\
\hline Archivística & Pobreza, medio ambiente y las luchas sociales. \\
\hline Ciencias Políticas & $\begin{array}{l}\text { Situación política del país, pobreza, ausencia de la educación y el } \\
\text { Tratado de Libre Comercio con Estados Unidos (TLC). }\end{array}$ \\
\hline $\begin{array}{c}\text { Ciencias de la } \\
\text { Comunicación* } \\
\text { Colectiva }\end{array}$ & $\begin{array}{l}\text { Pobreza, situación de la educación en el país, mala distribución de } \\
\text { la riqueza, violencia e inseguridad. }\end{array}$ \\
\hline $\begin{array}{c}\text { Trabajo Social } \\
\text { Sociología }\end{array}$ & $\begin{array}{l}\text { Situación política del país, mala distribución de la riqueza, la } \\
\text { condición de la educación y la pobreza. }\end{array}$ \\
\hline Pobreza, situación política, situación de la educación y violencia e \\
inseguridad.
\end{tabular}

Fuente: Esquivel, 2007, p. 214.

*Las personas encuestadas se identificaron únicamente como estudiantes de Ciencias de la Comunicación Colectiva, sin destacar énfasis de titulación. 
Como se evidencia, el tema de la pobreza tiene una importante prioridad en cinco de las siete carreras de dicha Facultad, seguido principalmente por la situación política del país.

\section{Cuadro $\mathrm{N}^{\circ} 2$}

Principales problemas sociales de importancia para la formación, según los estudiantes encuestados por carrera empadronada. Facultad de Educación UCR, 2007

\begin{tabular}{|c|l|}
\hline Carrera & \multicolumn{1}{|c|}{ Valoración } \\
\hline Enseñanza Primaria & $\begin{array}{l}\text { Violencia e inseguridad, pobreza, situación de la } \\
\text { educación, situación política y desempleo. }\end{array}$ \\
\hline Enseñanza de la Filosofía & Pobreza y mala distribución de la riqueza. \\
\hline $\begin{array}{c}\text { Enseñanza de las } \\
\text { Matemáticas }\end{array}$ & Pobreza, desempleo. \\
\hline Orientación educativa & Drogadicción y situación de la educación. \\
\hline Educación especial & Mala distribución de la riqueza y situación de la educación. \\
\hline $\begin{array}{c}\text { Enseñanza de los Estudios } \\
\text { Sociales }\end{array}$ & Pobreza, situación de la educación y desempleo. \\
\hline Enseñanza del castellano & Situación actual de la educación. \\
\hline Enseñanza de las Ciencias & Pobreza, situación de la educación. \\
\hline Enseñanza del Inglés & Desempleo e inseguridad. \\
\hline Enseñanza de la Música & $\begin{array}{l}\text { Situación política, mala distribución de la riqueza y la } \\
\text { pobreza. }\end{array}$ \\
\hline Fuente: msunver \\
\hline
\end{tabular}

Fuente: Esquivel, 2007, p. 214

(En algunas carreras se reciben aportes de diferentes Facultades, sin embargo, se parte de la Facultad en la que se debe empadronar el estudiante para titularse en la misma)

A diferencia de la anterior Facultad, en Educación la pobreza no tiene tanto peso prioritario, aunque sí se propone como tema de relevancia, antes que violencia e inseguridad, drogadicción, mala distribución de la riqueza, condición actual de la educación, el desempleo y la situación política del país. 


\section{Cuadro $N^{\circ} 3$}

\section{Principales problemas sociales de importancia para su formación, según los estudiantes encuestados por carrera. Facultad de Ciencias Económicas UCR, 2007}

\begin{tabular}{|c|l|}
\hline Carrera & \multicolumn{1}{|c|}{ Valoración general de la formación académica universitaria } \\
\hline $\begin{array}{c}\text { Dirección de } \\
\text { Empresas }\end{array}$ & $\begin{array}{l}\text { Desempleo, pobreza, mala distribución de la riqueza y situación } \\
\text { política del país. }\end{array}$ \\
\hline $\begin{array}{c}\text { Administración } \\
\text { Pública }\end{array}$ & Pobreza, desempleo, falta de educación. \\
\hline Estadística & Pobreza, la mala distribución de la riqueza y el desempleo. \\
\hline $\begin{array}{c}\text { Contaduría } \\
\text { Pública }\end{array}$ & Pobreza, desempleo y el TLC. \\
\hline Economía & Pobreza, desempleo, mala distribución de la riqueza. \\
\hline $\begin{array}{c}\text { Administración } \\
\text { Aduanera }\end{array}$ & Desempleo, pobreza, mala distribución de la riqueza. \\
\hline
\end{tabular}

Fuente: Esquivel, 2007, p. 215

En esta Facultad, el tema de la pobreza también aparece como prioritario en las personas de las carreras encuestadas, muy similar a Ciencias Sociales, y distinto a lo obtenido en las respuestas de la Facultad de Educación.

Finalmente, en lo que respecta a la Facultad de Derecho, entre los problemas sociales de relevancia, las personas encuestadas identifican la situación política, la violencia y la inseguridad.

Los datos arrojaron que el tema de la pobreza no es una prioridad como problema social de estudio para la gran mayoría de estudiantes encuestados de dicha Facultad.

De forma general, y según se ha expuesto, el tema de la pobreza, a partir del criterio de las personas encuestadas, sí es considerado relevante de abordar en la formación académica universitaria, aunque esa prioridad, puede variar por carrera y facultad.

Por lo tanto, queda evidencia de que la pobreza, es un eje de preocupación para las personas que participaron en la investigación, y por lo tanto, un desafío para la producción de conocimiento desde la investigación educativa universitaria, a pesar de que en el análisis de antecedentes de poca cuenta de ello (Ver Esquivel, 2007).

Hasta acá, se puede plantear que la pobreza en la priorización de problemas sociales, por parte de los estudiantes consultados, se ubica como un eje relevante, lo cual, evidencia la necesidad de discutir la manera en que se relaciona y fundamenta la incorporación de dicho tema en la formación académica universitaria. 
Recuérdese que otros problemas sociales que se señalan de importancia en la formación académica, según los estudiantes, son la situación política del país, la violencia e inseguridad, la drogadicción, la mala distribución de la riqueza, la condición actual de la economía y el desempleo.

En conclusión se puede afirmar que la temática de la pobreza, se torna una referencia significativa y primaria en el análisis del significado de las carreras universitarias y sus vínculos con la sociedad costarricense, justamente el siguiente apartado profundiza ese particular.

\section{La pobreza, sus causas e importancia en la formación académica}

Como se ha ido evidenciando en el desarrollo de la investigación, los recientes antecedentes académicos sobre el tema de la pobreza y su relevancia en la formación son limitados.

En el particular de la pobreza, 60 estudiantes encuestados del total, alegan haber llevado o tener que matricular algún curso que vincule su profesión con ese tema; ello significa que un $79 \%$ de las personas consultadas no identifican alguna materia que establezca esa relación; sobre esa interrogante se elaboró el siguiente gráfico:

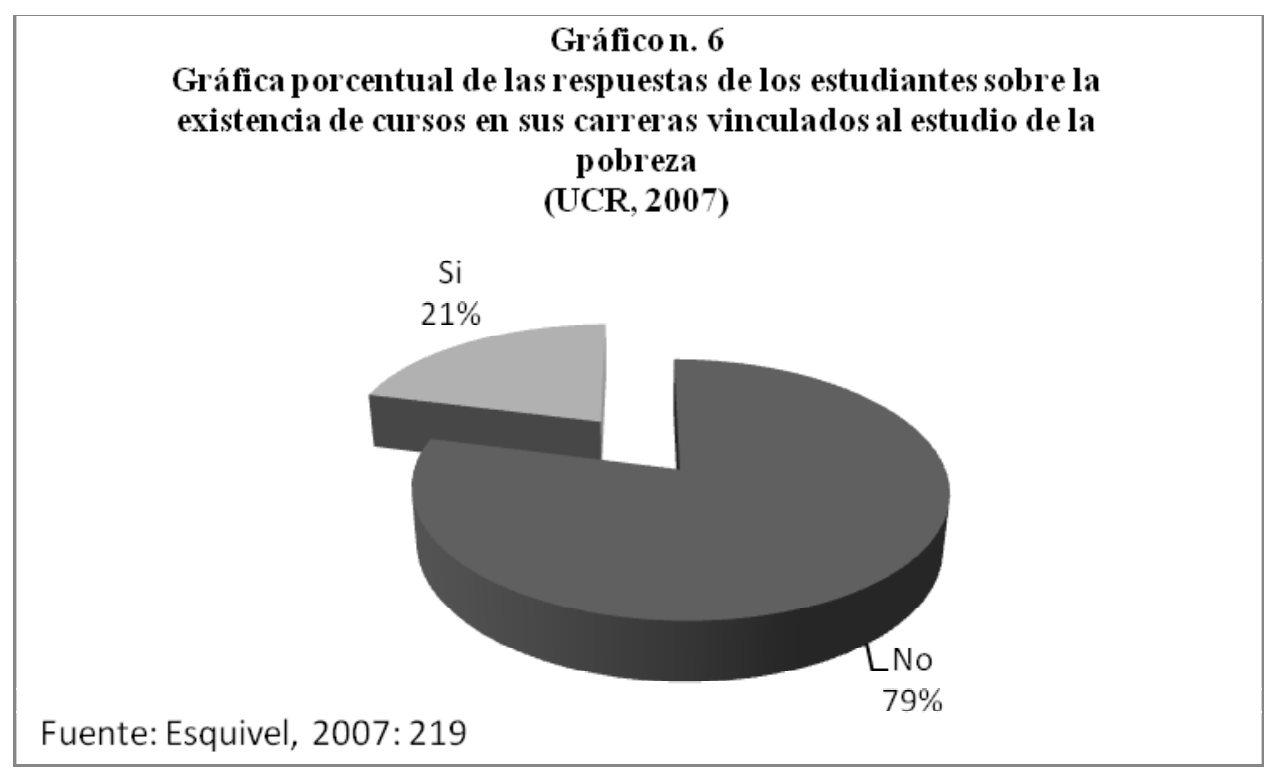

Las facultades a las que pertenecen las personas que afirmaron llevar algún curso vinculado al tema de la pobreza, se desglosan en el siguiente gráfico: 




A pesar de que un porcentaje tan limitado del total de estudiantes encuestados $(21 \%)$, afirma que ha llevado un curso vinculado al tema de la pobreza, un $76 \%$ de los estudiantes encuestados del total de facultades, asiente que su formación académica universitaria, ha influido en su explicación de la pobreza antes de entrar a la universidad; justamente para los universitarios consultados, las principales causas de la pobreza son las siguientes:

\section{Gráficon. 8}

Gráfica de distribución porcentual de las principales causas de la pobreza, según estudiantes encuestados.

(UCR, 2007)

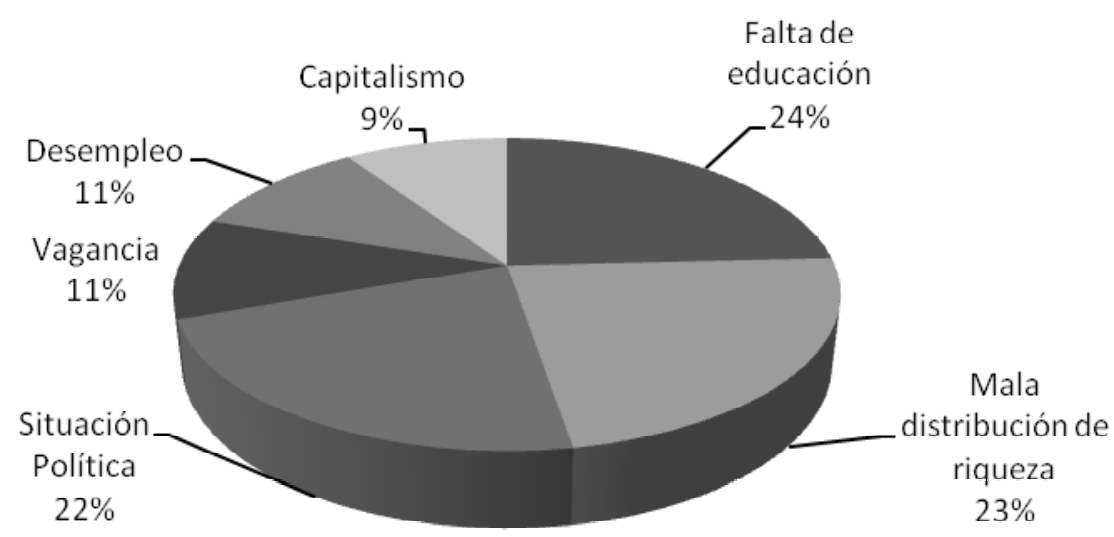

Fuente: Esquivel, 2007: 222 
De ser así, esa relación entre la formación y el "cambio" de explicación de la pobreza, parece predominar en hallar su naturaleza en la ausencia de altos niveles educativos formales, mala distribución de la riqueza y la situación política nacional.

Sin embargo, en este artículo se hace una ruptura sobre ese argumento predominante, ya que a juicio de quien escribe, el origen de la pobreza no radica en el plano educativo ${ }^{12}$, sino que predomina en la producción y distribución social de la riqueza, por ende, en las relaciones de la naturaleza fundamental del capitalismo (Por tanto, se apoya la causa que prevalece en las respuestas dadas por los estudiantes de Psicología y Sociología, ver cuadro N.4)

Por otra parte, la investigación también posibilitó identificar, que entre las carreras existen sus singularidades sobre las explicaciones de la causalidad de la pobreza, los siguientes datos ilustran esas diferencias:

12 Lo anterior se fundamenta, en que según Córdoba (2007), en Centroamérica y República, Dominicana, con excepción de Costa Rica, quienes tienen más títulos, son las personas que más dificultades enfrentan para encontrar empleo. Por ejemplo, un hombre que tenga cursada la secundaria, tiene un $10 \%$ de probabilidades de no conseguir empleo, y un $15 \%$ una mujer; mientras que para las personas con sólo primaria, se reduce en un $5 \%$ a su favor esa probabilidad, según el sexo $(10 \%$ y $5 \%$, respectivamente). Sólo en el caso de las mujeres, la educación sí parece estar mediando en las oportunidades de trabajo, pues únicamente un $30 \%$ de las mujeres sin educación, forman parte de la fuerza laboral de la región, mientras que la tasa de participación para quienes tienen educación superior, llega a un $70 \%$. Para Córdoba, la economía centroamericana, aún mantiene poca capacidad de adaptación a tecnologías y procesos productivos de rápida transformación; sumado a ello se localiza una diversidad muy escasa y obsoleta; por ejemplo, un $80 \%$ de los ocupados en agricultura, aún recurre a técnicas de cultivo tradicionales. Por lo tanto, se propone que la educación no sólo debe mejorar la calificación de la mano de obra, sino también estimular el interés de la inversión, debido a sus habilidades, destrezas y conocimientos. Vinculado lo anterior, a trabajos que incluyan ingreso justo, seguridad, y protección social para las familias. En términos generales, en la región, el $42 \%$ de las personas no ha logrado terminar la primaria (de ellas un $61 \%$ en las zonas rurales), mientras que el $13 \%$ de la población centroamericana, nunca inició educación alguna. A su vez, la empresa privada regional, sólo emplea al $45 \%$ de las personas ocupadas, por lo que hay un escaso desarrollo del empleo asalariado, y por ende un incremento en el trabajo informal. El istmo, tiene un $62 \%$ de población ocupada con salario patronal en las ciudades, y un $42 \%$ en el área rural; la agricultura concentra un $27 \%$ de los ocupados, y los servicios un $25 \%$. Provocando también una media de desempleo de $7,9 \%$ para la región. (Sólo dos de cada cinco mujeres en edad activa, tiene un empleo)El Ilamado "autoempleo", que incluye a trabajadores por cuenta propia o familiares no remunerados, se convirtió en la forma de inserción laboral para dos quintas partes de los ocupados que están trabajando en la región. 


\section{Cuadro $\mathrm{N}^{\circ} 4$}

Principales causas de la pobreza, según estudiantes encuestados por carrera.

Facultad de Ciencias Sociales.

UCR, 2007

\begin{tabular}{|c|c|c|}
\hline Carrera & $\begin{array}{l}\text { Cantidad de } \\
\text { estudiantes }\end{array}$ & $\begin{array}{c}\text { Causas según cantidad de personas } \\
\text { que la indicaron }\end{array}$ \\
\hline Antropología & 12 & $\begin{array}{l}\text { Mala distribución de la riqueza (7) } \\
\text { Situación política del país (5) }\end{array}$ \\
\hline Archivo Administrativo & 2 & $\begin{array}{l}\text { Mala distribución de la riqueza (1) } \\
\text { Situación política del país (1) }\end{array}$ \\
\hline Ciencias Políticas & 7 & $\begin{array}{c}\text { Capitalismo (5) } \\
\text { Situación política del país (2) }\end{array}$ \\
\hline Comunicación Colectiva & 9 & $\begin{array}{c}\text { Mala distribución de la riqueza (3) } \\
\text { Capitalismo (2) } \\
\text { Falta de educación (2) } \\
\text { Vagancia (1) } \\
\text { Desempleo (1) }\end{array}$ \\
\hline Trabajo Social & 12 & $\begin{array}{c}\text { Ausencia de educación (5) } \\
\text { Mala distribución de la riqueza (3) } \\
\text { Situación política (2) }\end{array}$ \\
\hline Sociología & 15 & $\begin{array}{c}\text { Mala distribución de la riqueza (5) } \\
\text { Situación política (4) } \\
\text { Falta de educación (3) } \\
\text { Falta de empleo (3) }\end{array}$ \\
\hline Psicología & 12 & $\begin{array}{c}\text { Capitalismo (5) } \\
\text { Falta de educación (3) } \\
\text { Situación política (3) } \\
\text { Mala distribución riqueza (3) }\end{array}$ \\
\hline Historia & 3 & $\begin{array}{l}\text { Mala distribución de la riqueza (2) } \\
\text { Falta de educación (1) }\end{array}$ \\
\hline Geografía & 3 & $\begin{array}{l}\text { Mala distribución de la riqueza (2) } \\
\text { Falta de educación (2) }\end{array}$ \\
\hline Total & 75 & \\
\hline
\end{tabular}

Fuente: Esquivel, 2007, p. 230 


\section{Cuadro $N^{\circ} 5$}

Principales causas de la pobreza, según estudiantes encuestados por carrera. Facultad de Educación UCR, 2007

\begin{tabular}{|c|c|c|}
\hline Carrera & $\begin{array}{l}\text { Cantidad de } \\
\text { estudiantes }\end{array}$ & $\begin{array}{c}\text { Causas según cantidad de personas } \\
\text { que la indicaron }\end{array}$ \\
\hline Educación Primaria & 19 & $\begin{array}{c}\text { Falta de educación (7) } \\
\text { Situación política del país (2) } \\
\text { Mala distribución de la riqueza (2) } \\
\text { Desempleo (3) } \\
\text { Vagancia (3) }\end{array}$ \\
\hline Orientación & 2 & $\begin{array}{l}\text { Falta de educación (1) } \\
\text { Situación política del país (1) }\end{array}$ \\
\hline Educación Especial & 2 & Situación política del país (2) \\
\hline $\begin{array}{l}\text { Enseñanza de las } \\
\text { Matemáticas }\end{array}$ & 8 & $\begin{array}{l}\text { Falta de educación (3) } \\
\text { Mala distribución de riqueza (3) } \\
\text { Situación política del país (2) }\end{array}$ \\
\hline $\begin{array}{c}\text { Enseñanza de las Ciencias } \\
\text { Naturales }\end{array}$ & 14 & $\begin{array}{c}\text { Falta de educación (7) } \\
\text { Situación política del país (4) } \\
\text { Mala distribución de riqueza (3) }\end{array}$ \\
\hline $\begin{array}{l}\text { Enseñanza de los Estudios } \\
\text { Sociales }\end{array}$ & 25 & $\begin{array}{c}\text { Vagancia (10) } \\
\text { Situación política del país (6) } \\
\text { Desempleo (5) } \\
\text { Capitalismo (4) }\end{array}$ \\
\hline Enseñanza del Francés & 6 & $\begin{array}{l}\text { Falta de educación (3) } \\
\text { Situación política (3) }\end{array}$ \\
\hline Enseñanza del Inglés & 4 & $\begin{array}{l}\text { Falta de educación (3) } \\
\text { Vagancia (1) }\end{array}$ \\
\hline Enseñanza de la Música & 4 & Falta de educación (4) \\
\hline Enseñanza del Castellano & 2 & $\begin{array}{l}\text { Falta de educación (1) } \\
\text { Situación política (1) }\end{array}$ \\
\hline Enseñanza de la Filosofía & 2 & $\begin{array}{l}\text { Falta de educación (1) } \\
\text { Situación política (1) }\end{array}$ \\
\hline Total & 88 & \\
\hline
\end{tabular}

Fuente: Esquivel, 2007, p. 231 


\section{Cuadro $N^{\circ} 6$}

Principales causas de la pobreza, según estudiantes encuestados.

Facultad de Derecho

UCR, 2007

\begin{tabular}{|c|c|c|}
\hline Carrera & $\begin{array}{c}\text { Cantidad de } \\
\text { estudiantes }\end{array}$ & $\begin{array}{c}\text { Causas según cantidad de personas } \\
\text { que la indicaron }\end{array}$ \\
\hline Derecho & 34 & $\begin{array}{c}\text { Falta de educación (20) } \\
\text { Situación política del país (7) } \\
\text { Mala distribución de la riqueza (7) }\end{array}$ \\
\hline
\end{tabular}

Fuente: Esquivel, 2007, p. 231

\section{Cuadro $\mathrm{N}^{\circ} 7$}

Principales causas de la pobreza, según estudiantes encuestados por carrera. Facultad de Ciencias Económicas

UCR, 2007

\begin{tabular}{|c|c|c|}
\hline Carrera & $\begin{array}{c}\text { Cantidad de } \\
\text { estudiantes }\end{array}$ & $\begin{array}{c}\text { Causas según cantidad de personas } \\
\text { que la indicaron }\end{array}$ \\
\hline Dirección de empresas & 32 & $\begin{array}{c}\text { Falta de educación (25) } \\
\text { Situación política del país (7) }\end{array}$ \\
\hline Contaduría Pública & 40 & $\begin{array}{c}\text { Falta de educación (20) } \\
\text { Distribución de la riqueza (13) } \\
\text { Situación política del país (7) }\end{array}$ \\
\hline Administración Pública & 10 & $\begin{array}{c}\text { Falta de educación (5) } \\
\text { Situación política del país (5) }\end{array}$ \\
\hline Administración Aduanera & 7 & $\begin{array}{c}\text { Falta de educación (4) } \\
\text { Situación política (3) }\end{array}$ \\
\hline Economía & 5 & $\begin{array}{c}\text { Falta de educación (2) } \\
\text { Situación política del país (2) } \\
\text { Desempleo (1) }\end{array}$ \\
\hline Estadística & 1 & Falta de educación (1) \\
\hline Total & 95 & \multicolumn{2}{|c}{}
\end{tabular}

Fuente: Esquivel, 2007, p. 232

Para el caso de los docentes encuestados, la pobreza tiene una importancia "regular" en la formación académica de la UCR, el siguiente gráfico detalla esa información: 


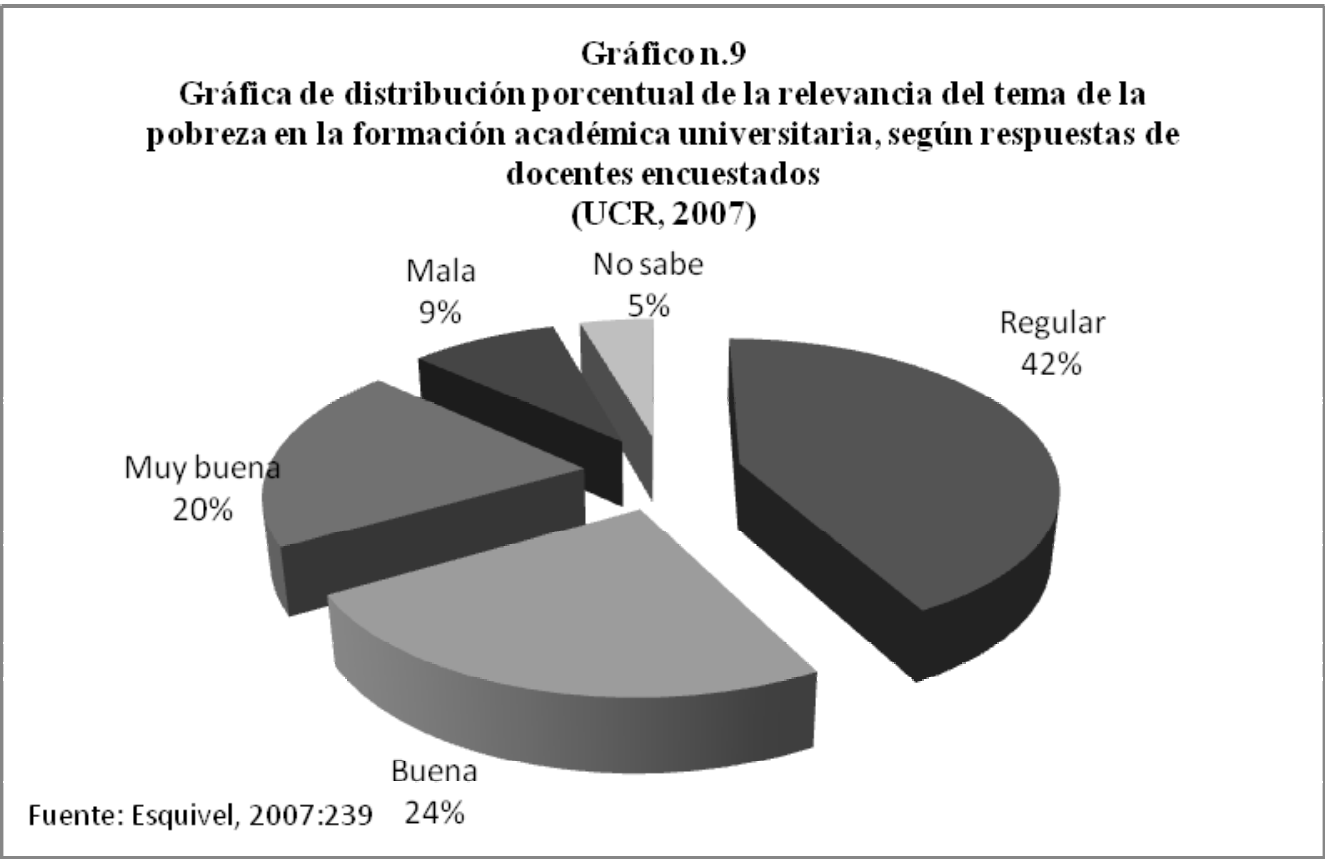

Para los académicos en consulta, las principales causas de la pobreza se expresan en el siguiente gráfico:

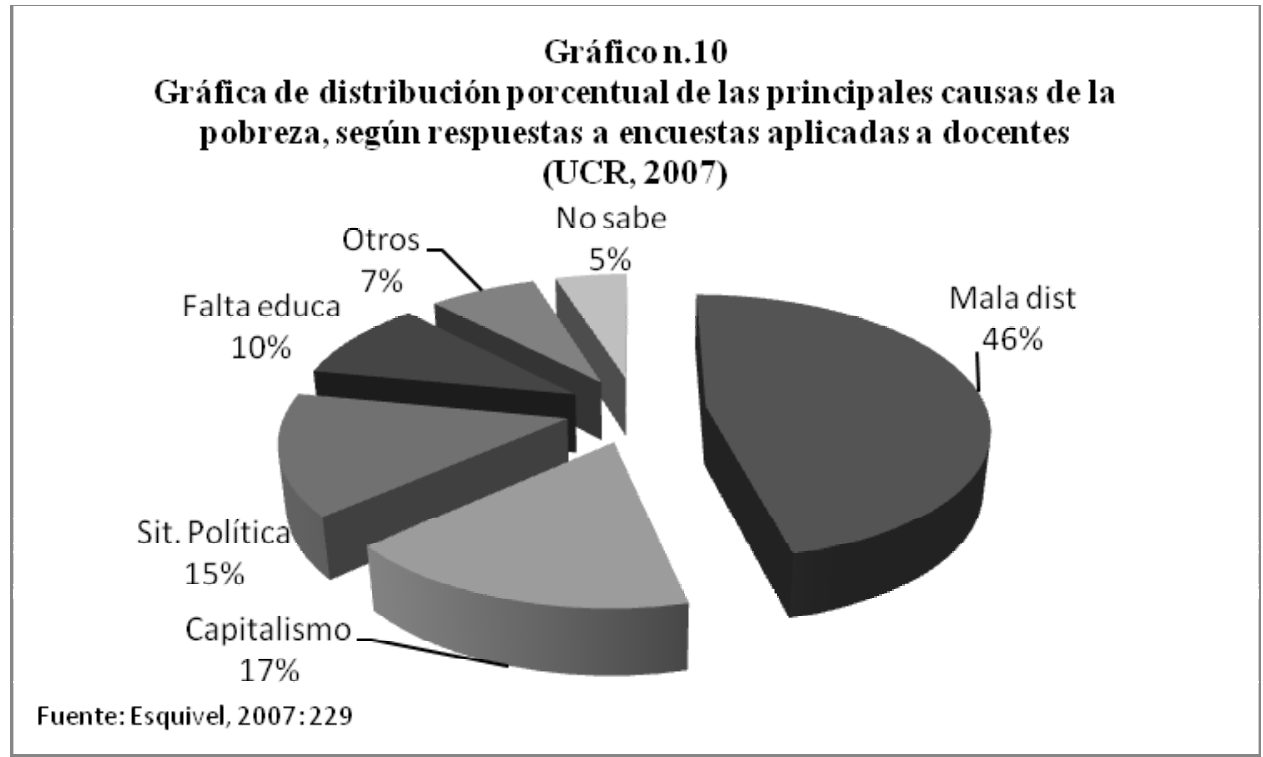

De los datos antes obtenidos, se expresa una diferencia con los criterios de los estudiantes, donde los académicos colocan el tema de la ausencia educativa como cuarta causa de la pobreza. 
En síntesis y partiendo de los datos antes expuestos, es posible meditar que la pobreza, según la mayoría de los estudiantes consultados, se podría considerar como un tema significativo en los cursos de sus planes de estudio. Por su lado para los docentes, la pobreza es un tema de relevancia regular en la formación académica universitaria en general.

Aunque la pobreza se puede identificar por parte de los estudiantes, como un tema de relevancia para la formación académica universitaria (con los cuidados críticos del caso), no se localiza una explicación que supere el tradicional argumento académico y político, que su respuesta se encuentra en elevar los niveles educativos.

A pesar de ello, la formación académica universitaria, ha cambiado las explicaciones de la pobreza para la mayoría de las personas encuestadas (lo cual no significa, que se aborde desde una visión crítica o bien en contraposición con el discurso antes generalizado sobre la "naturaleza" de la pobreza).

\section{6- Conclusiones y recomendaciones:}

El presente estudio, permitió ilustrar y generar algunos argumentos para la reflexión sobre la importancia del tema de los problemas sociales, y en especial de la pobreza en la formación académica de la UCR.

La necesidad de esta interrogación, tal y como se ha recordado, surge por la imperiosa necesidad del significado que adquiere la educación superior pública ante el contexto nacional, aunado a los impulsos teleológicos que fundamentan el Estatuto Orgánico de esta alma máter.

Los datos antes expuestos permiten argumentar que el tema de la formación académica debe recibir mayor atención investigativa, especialmente en lo que a sus relaciones contextuales y coyunturales refiere, ejemplificado en este caso en el estudio de los llamados problemas sociales y la pobreza.

En ese particular, este último tema demostró adquirir un significado relevante entre las prioridades que los estudiantes colocan como ámbito de interés para sus estudios, ello a pesar de la poca relación que reconocen de ese tema en sus planes de estudio.

La causa de la pobreza, no necesariamente fue definida de igual manera por los estudiantes y docentes; en los primeros la respuesta que prevalece, es que ella es resultado 
de la ausencia de educación formal, y en los segundos se localiza el argumento de la mala distribución de la riqueza.

Al tomar los datos por facultades, y carreras, las explicaciones manifiestan otras naturalezas; por ello, puede plantearse la conjetura, de que la formación académica, según el área de pertenencia del estudiante (sea de Educación, Ciencias Sociales, Ciencias Económicas o Derecho), incide en los argumentos que las personas brindan para explicar el tema de la pobreza, sea como "problemas legales, educativos, sociales y económicos".

Lo anterior merece mayor investigación, sin embargo, es posible afirmar que la mayoría de las personas encuestadas, reconocen de todas maneras, que la pobreza no ha sido un tema relevante en los cursos de su formación; sin embargo, aceptaron, en un porcentaje bastante significativo, que los estudios universitarios variaron sus tradicionales explicaciones de la misma.

Finalmente, se considera que una sugerencia importante que surge del análisis realizado, es la necesidad de proponer como línea de investigación, quizás al amparo de la infraestructura del Instituto de Investigación Educativa de la UCR (INIE), el tema Educación y "Cuestión Social", en tanto evidencia ser un área prometedora y problematizadora de la producción de conocimientos en educación.

Dentro de la línea de investigación antes sugerida, sería conveniente establecer un componente que aborde de manera privilegiada el tema de la pobreza, en tanto pueda ser cuestionada como una expresión de importante envergadura para la discusión educativa nacional y latinoamericana, y a su vez, haga ruptura con bastantes tesis explicativas que abstraen la pobreza de la "cuestión social".

\section{Referencias}

Córdoba, Javier. (2005, 14 de junio). Más educación no garantiza trabajo en Centroamérica. San José, Costa Rica. Periódico Semanario Universidad, p. 10. Universidad de Costa Rica.

Elejabarrieta, F. e Iguiñez, Lupicinio. (1984). Construcción de Escalas. Buenos Aires, Argentina: UBA.

Esquivel, Freddy. (2007) Análisis de las contradicciones entre la formación académica de la Universidad de Costa Rica y las bases orgánico institucionales que la vinculan con el tema de la pobreza. Tesis de posgrado para optar por el título de Doctor en Educación. Sistema de Estudios de Posgrado. Programa de Doctorado Latinoamericano en Educación. Universidad Estatal a Distancia. San José, Costa Rica. 
Hernández, Roberto, et al., (1998). Metodología de la investigación. México: McGraw-Hill Interamericana Editores, SA.

Mészáros, István. (1998). Para ir más allá del capital. Recuperado el 2 octubre 2005, de www.herramientas.com.ar/varios

Proyecto Estado de la Nación. (2007). XIII Informe Estado de la Nación en Desarrollo Humano Sostenible. San José, Costa Rica: Programa Estado de la Nación (Costa Rica).

Real Academia Española. (2001). Diccionario Enciclopédico. Barcelona, España: Ediciones Luz.

Rojas, Elizabeth. (2005). Centroamérica en la mira de los economistas. San José, Costa Rica. Semanario Universidad, Suplemento Crisol, p. 3.

Salazar, Jorge. (1995). Crisis liberal y estado reformista. Análisis político electoral. San José, Costa Rica: EUCR.

Solís, Manuel. (1992). Costa Rica: ¿Reformismo social demócrata o liberal? FLACSO: San José, Costa Rica.

Universidad de Costa Rica. (2006). Guía de Matricula de Trabajo Comunal Universitario. San José, Costa Rica: Vicerrectoría de Acción Social.

Universidad de Costa Rica. (2007). Estatuto Orgánico. San José, Costa Rica: Oficina de Publicaciones de la Universidad de Costa Rica.

Vargas, Luis. (2006). Entre la vida y el mercado. Tratado de Libre Comercio Estados Unidos-Centroamérica-República Dominicana. (TLC-EDUCARD). Implicaciones para la educación. San José, Costa Rica: EUNED. 